\title{
Yearly Suicides across Canada, Great Britain and the United States from 1960 to 2015: A Search for Underlying Long-Term Trends
}

\author{
William Henry Laverty ${ }^{1}$, Ivan William Kelly² \\ ${ }^{1}$ Department of Mathematics and Statistics, University of Saskatchewan, Saskatoon, Canada \\ ${ }^{2}$ Department of Educational Psychology \& Special Education, University of Saskatchewan, Saskatoon, Canada \\ Email: laverty@math.usask.ca
}

How to cite this paper: Laverty, W.H. and Kelly, I.W. (2019) Yearly Suicides across Canada, Great Britain and the United States from 1960 to 2015: A Search for Underlying Long-Term Trends. Open Journal of Social Sciences, 7, 107-115.

https://doi.org/10.4236/jss.2019.76008

Received: May 9, 2019

Accepted: June 16, 2019

Published: June 19, 2019

Copyright () 2019 by author(s) and Scientific Research Publishing Inc. This work is licensed under the Creative Commons Attribution International License (CC BY 4.0).

http://creativecommons.org/licenses/by/4.0/

\begin{abstract}
Suicide is a serious social problem across the world. In this article we examine suicide rates in three Western countries over a longer period of time than is typically considered to determine if extended underlying patterns are discernable. Since assumptions of traditional time series analyses are likely violated with such long-term data, we have utilized Hidden Markov probability models in analyzing yearly suicide data (including birth sex) over a 60-year period across three countries (Canada, USA, and Great Britain). Apart from the expected larger numbers of suicides by males across all three countries, we uncovered two underlying states of 25.39 and 12.61 years duration within which differing trends for males and females are evident.
\end{abstract}

\section{Keywords}

Suicide, Yearly Suicide Totals, Hidden Markov Model, Autoregressive HMM, Suicide in Great Britain, Suicide in Canada, Suicide in the United States

\section{Introduction}

The World Health Organization (WHO) estimates that more than three-quarters of a million people worldwide take their own lives yearly [1]. The majority of suicides occur in low and middle income countries, and suicide is the second leading cause of death worldwide for individuals in the 15 - 29 age range. Previous studies have indicted suicide outcomes to be related to occupation and levels of job stress. Milner, Spittal, Pirkis and Lamontagne [2] conducted a meta-analysis on 34 studies on suicide across occupations and found that the lowest skill occupations were overall at higher risk of suicide than those working in higher skill 
occupations. Part of the reason for such differences may be due to lower skill occupations being associated with higher job stress. In support of this conjecture, Milner, Witt, LaMontagne, and Niedhammer [3] meta-analyzed 22 studies that considered job stressors and suicide and found that lower supervisor and colleague support (related to higher job stress) were related to higher suicidal outcomes. At the same time, at particular times in higher social class careers, suicide rates can be associated with different kinds of stressors and high suicide rates. An example of the latter can be found in student and resident rates in medical school [4].

The three countries chosen for inclusion in this study (Canada, The United States, and Great Britain) were done so for their overlapping histories, common central language, political and economic traditions, and data accessibility. In the United Kingdom (Great Britain), the standardized suicide rate (in 2017) was 10 deaths per 100,000 people, with the highest rate among males aged 45 to 49 , and aged 50 to 54 for females. The rate for males was, overall, three times that of females [5]. At the same time, there has been a decrease in all overall suicide rate in male suicide. In the UK, there has been a significant decrease in male suicide. The male suicide rate in the years 2016-2017 was the lowest in over 30 years. In Scotland the overall suicide rate decreased during 2016-2017 but remained stable overall in Northern Ireland over the same time period [6].

In Canada, according to the Canadian Association for Suicide Prevention (CARP) [7],

Males are three times likely to die by suicide than females, with higher rates prevailing at a consistent trend. Although men are more likely to die by suicide, females are three times more likely to attempt to end their lives. In addition, women are hospitalized 1.5 times more often than males for suicide related behaviors. The discrepancy may be due to the fact that females tend to use less immediately lethal methods. Based on the Canadian Community Health Survey, $14.7 \%$ of Canadians have thoughts about suicide and 3.5\% have attempted suicide in their lifetime. (Assessed May 3, 2019)

The most at-risk Canadian group for suicide is those in the age range 15 - 24 . In this Canadian age group, severe depression and suicidal thoughts were considered characteristics of suicidal behavior [8].

In the United States, the American Foundation for Suicide Prevention [9] [10] informs us that suicide is the $10^{\text {th }}$ leading cause of death in that country, with over 43,000 Americans taking their own lives in the year 2017 alone (assessed May 3, 2019). In 2017, men died by suicide more than three times the rate for women.

\section{Methodology}

\subsection{Data Collection}

The suicide data were obtained from the OECD database [11]. 
Suicide rates are defined as the deaths deliberately initiated and performed by a person in the full knowledge or expectation of its fatal outcome. Comparability of data between countries is affected by a number of reporting criteria, including how a person's intention of killing themselves is ascertained, who is responsible for completing the death certificate, whether a forensic investigation is carried out, and the provisions for confidentiality of the cause of death. Caution is required therefore in interpreting variations across countries. The rates have been directly age-standardized to the 2010 OECD population to remove variations arising from differences in age structures across countries and over time. The original source of the data is the WHO Mortality Database. This indicator is presented as a total and per gender and is measured in terms of deaths per 100,000 inhabitants (total), per 100,000 men and per 100,000 women.

Different kinds of information can be obtained depending on the time frame considered in the study (daily, weekly, monthly, yearly, decade) and the type and number of variables used in the study (sex, age, SES, location, weather conditions, etc). While each study can utilize different time units and variables, each approach can contribute in different ways to an understanding of suicide. In this study we have chosen to analyze data using yearly total suicides in three Western countries (USA, UK, and Canada) over the time period 1960-2015. The yearly data was differenced to obtain yearly changes in suicide rates, resulting in data that is approximately stationary in nature. The birth sex of the suicides was included as clear patterns of differencing the sexes have been uniformly found across the world, and consequently, we were interested in determining possible different underlying trends in suicides by sex.

\subsection{Analysis Method}

Traditionally, in social research, longitudinal studies have been analyzed using time series methods (ARMA, ARIMA etc.). The random component that is driving these models is assumed to have constant distributional properties. This is not always true. If the data is observed over longer periods of time there are likely underlying states generating the observed data with differing distributional properties. In this case it is important that the differing underlying hidden states are identified. These hidden states would correspond to periods where underlying social, political, or environmental factors have changed. To uncover possible underlying states Hidden Markov and Autoregressive Hidden Markov models were fit to the data in this study. The data analyzed was yearly changes rather than the raw data to obtain some level of stationarity (the distribution is time independent).

\section{Results}

\subsection{Descriptive Statistics}

The mean suicide rates for the six groups (Canada-Male, Female; Great Brit- 
ain-Male, Female; US-Male, Female) over the 60 year period are in Table 1.

The table above indicates that over the long time period under consideration, the male rate of suicide was three times that of females in both the Canada and the United States, and over twice the rate in Great Britain.

The graph of the raw data (no differencing) in Figure 1 shows the suicide rates for the three countries separated according to sex over the sixty-year time period.

The graph (Figure 1) and table (Table 1) reinforces the expected finding of a consistent pattern of higher male suicides than female over time in all three countries. Males in all three countries also show more volatility over time than females. This graph also indicates when the suicide rates for specific groups are increasing or decreasing over time.

The graph (Figure 2) shows volatility in the year to year changes in suicide rates. Without further analysis it is difficult to assess whether the central values of the yearly changes in suicide rates are nonzero (positive or negative) during particular periods of time. During positive average changes the suicide rate is increasing and during negative average changes the rate is decreasing. It will be noted that the greatest volatility is shown in yearly changes in Canadian males. However, males in all three countries show greater volitivity in changes in suicide than females.

Table 1. Mean suicide rates birth sex for Canada, Great Britain and the United States.

\begin{tabular}{cccccc}
\hline \multicolumn{2}{c}{ Canada } & \multicolumn{2}{c}{ Great Britain } & \multicolumn{2}{c}{ US } \\
\hline Male & Female & Male & Female & Male & Female \\
\hline 19.76 & 6.06 & 12.06 & 5.15 & 21.01 & 5.79 \\
\hline
\end{tabular}

\section{Suicide Rates}

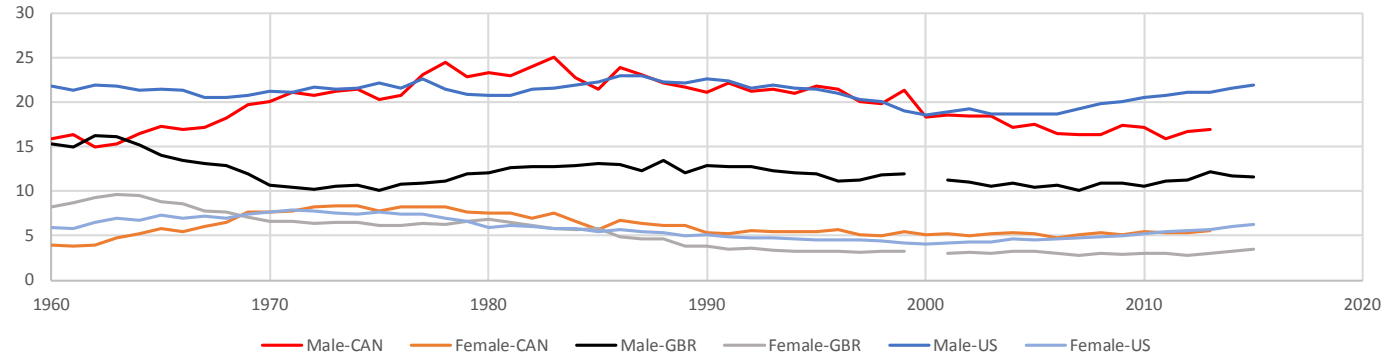

Figure 1. Raw data of suicide rates for birth sex and country over 60 years.

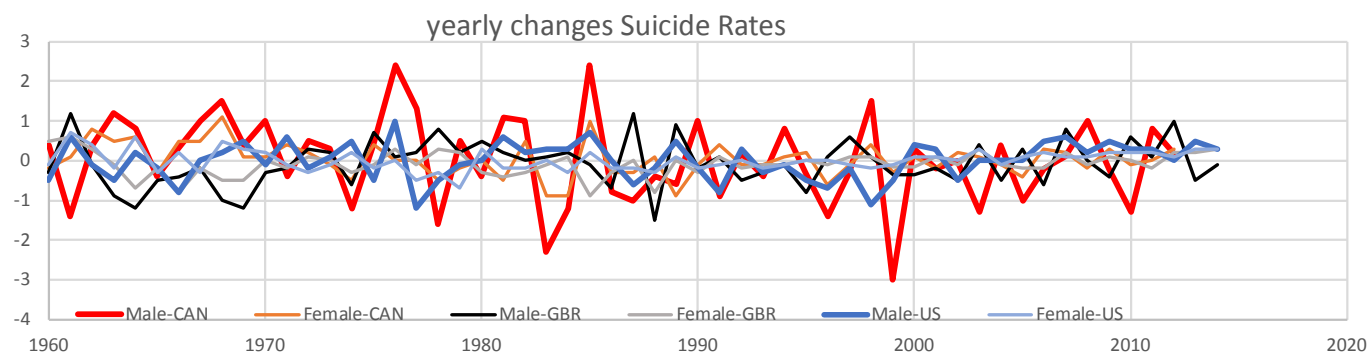

Figure 2. Yearly changes in suicide rate for sex and country over 60 years. 


\subsection{Data Analysis by HMM}

The authors initially considered modeling the data with an Autoregressive Hidden Markov model (ARHMM). This model allows for autocorrelation between the observation vectors when the process is in a given state. On the other hand, the simpler Hidden Markov Model (HMM) assumes that the observation vectors are independent when the process is in a given state. Once both models have been fit a test can be carried out to see whether the more advanced Autoregressive Hidden Markov Model is required over the simpler Hidden Markov model (HMM). The statistical test used is the asymptotic Likelihood Ratio test where the test statistic is: $U=2\left(\ln \left(L_{2}\right)-\ln \left(L_{1}\right)\right)$ which has a chi-square distribution with $v=$ degrees of freedom $=$ number of parameters of the ARHMM-number of parameters of the HMM $=42, \ln \left(L_{2}\right)=$ loglikelihood when fitting ARHMM and $\ln \left(L_{1}\right)=$ loglikelihood when fitting HMM [12]. When the models were fit it was found that $\ln \left(L_{2}\right)=-123.1061$ and $\ln \left(L_{1}\right)=-138.4736$.

Thus $U=30.735$ for $v=42$ degrees of freedom, $p$-value $=0.900716$, indicating that the ARHMM is not required over the HMM. Consequently, the following Parameter estimates reported are only for the HMM. In the fitting of the model two states were identified.

The first thing one notes in Table 2 is that the standard deviations are all large compared to their respective means, indicating much volatility within each country and between the sex of the suicide. In both states, positive means of yearly changes indicate the suicide rate is a random walk that is drifting upwards (increasing) while negative means indicate suicide rate is a random walk that is drifting downwards (decreasing). The means of yearly changes close to zero indicate behaviour of a stationary random walk. The above summary of the two fitted states indicates that when in state one there is a positive mean change for Canadian males. In other words, when the suicide rate for the three countries is in state one, the suicide rate for Canadian males is increasing (and for Canadian females as well, but less so), but decreasing for both males and females in Great Britain and the United States (note the rates of decrease are greater in Great Britain than the United States). In the second state, the suicide rate for males in all three countries is decreasing, with the rate of decrease higher in Canadian males. The suicide rate changes for females in state two is slight in all three countries.

Table 2. Summary of state parameters for the fitted HMM.

\begin{tabular}{cccccccc}
\hline & & \multicolumn{2}{c}{ Can } & \multicolumn{2}{c}{ GBR } & \multicolumn{2}{c}{ US } \\
\cline { 3 - 8 } & & Male & Female & Male & Female & Male & Female \\
\hline \multirow{2}{*}{ State 1 } & Mean & 0.203 & 0.043 & -0.079 & -0.161 & -0.002 & -0.027 \\
& Std Dev & 1.170 & 0.547 & 0.715 & 0.392 & 0.508 & 0.341 \\
\multirow{2}{*}{ State 2 } & Mean & -0.153 & 0.018 & -0.039 & -0.040 & -0.024 & 0.018 \\
& Std Dev & 0.938 & 0.265 & 0.450 & 0.152 & 0.440 & 0.153 \\
\hline
\end{tabular}


The correlation matrix (and Principle Components) of both states given (Table 3) provide information on the combined behavior of the suicide rates (and yearly changes) when the process is in that state. These provide information on what is happening within the states beyond that explained by the mean/average changes.

The principal components help us understand complex correlation structures. The first principal component is a linear combination of the observations that accounts for the maximum percentage of variance in the observations. When in state 1 , the first principal component indicates $63 \%$ of the variability of yearly suicide changes when in state 1 . A plausible interpretation of the loadings is that during state 1 increases in suicide rates in Great Britain correspond to decreases in Canada and the US and vice-versa.

When in state 2 (Table 4), the first principal component indicates $46 \%$ of the variability of yearly suicide changes when in state 1 . Similar to state 1 , interpretation of the loadings is that during state 2 increases in suicide rates in Great Britain correspond to decreases in Canada and the US and vice-versa.

Table 3. Correlation matrices and principle components for state 1. (a) Correlation matrix; (b) Principle Components.

(a)

\begin{tabular}{cccccccc}
\hline & & \multicolumn{2}{c}{ Can } & \multicolumn{2}{c}{ GBR } & \multicolumn{2}{c}{ US } \\
\cline { 3 - 8 } & & Male & Female & Male & Female & Male & Female \\
\hline \multirow{3}{*}{ Can } & Male & 1.000 & 0.662 & -0.338 & -0.310 & 0.129 & 0.032 \\
& Female & 0.662 & 1.000 & -0.405 & -0.395 & -0.055 & 0.419 \\
\multirow{2}{*}{ GBR } & Male & -0.338 & -0.405 & 1.000 & 0.592 & -0.003 & -0.103 \\
& Female & -0.310 & -0.395 & 0.592 & 1.000 & -0.034 & -0.139 \\
\multirow{2}{*}{ US } & Male & 0.129 & -0.055 & -0.003 & -0.034 & 1.000 & 0.418 \\
& Female & 0.032 & 0.419 & -0.103 & -0.139 & 0.418 & 1.000 \\
\hline
\end{tabular}

(b)

\begin{tabular}{|c|c|c|c|c|c|c|c|}
\hline & & \multicolumn{6}{|c|}{ Principle components } \\
\hline & & 1 & 2 & 3 & 4 & 5 & 6 \\
\hline \multirow{2}{*}{ Can } & Male & -0.433 & 0.516 & 0.241 & -0.072 & 0.021 & 0.695 \\
\hline & Female & -0.442 & 0.220 & 0.488 & -0.002 & -0.402 & -0.597 \\
\hline \multirow{2}{*}{ GBR } & Male & 0.403 & 0.215 & 0.363 & 0.810 & 0.040 & 0.049 \\
\hline & Female & 0.459 & 0.272 & -0.177 & -0.191 & -0.791 & 0.150 \\
\hline \multirow{2}{*}{ US } & Male & -0.311 & 0.419 & -0.732 & 0.384 & -0.005 & -0.211 \\
\hline & Female & -0.384 & -0.625 & -0.072 & 0.393 & -0.459 & 0.303 \\
\hline \multicolumn{2}{|c|}{ eigenvalue } & 3.775 & 0.888 & 0.792 & 0.369 & 0.162 & 0.014 \\
\hline \multicolumn{2}{|c|}{$\%$} & $62.9 \%$ & $14.8 \%$ & $13.2 \%$ & $6.1 \%$ & $2.7 \%$ & $0.2 \%$ \\
\hline \multicolumn{2}{|c|}{ cum $\%$} & $62.9 \%$ & $77.7 \%$ & $90.9 \%$ & $97.1 \%$ & $99.8 \%$ & $100.0 \%$ \\
\hline
\end{tabular}


Table 4. Correlation matrices and principle components for state 2. (a) Correlation matrix; (b) Principle Components.

(a)

\begin{tabular}{cccccccc}
\hline & & \multicolumn{2}{c}{ Can } & \multicolumn{2}{c}{ GBR } & \multicolumn{2}{c}{ US } \\
\cline { 3 - 8 } & & Male & Female & Male & Female & Male & Female \\
\hline \multirow{2}{*}{ Can } & Male & 1.000 & 0.409 & 0.000 & 0.030 & 0.057 & -0.175 \\
& Female & 0.409 & 1.000 & 0.057 & 0.410 & -0.024 & -0.097 \\
& Male & 0.000 & 0.057 & 1.000 & 0.583 & -0.006 & 0.036 \\
GBR & Female & 0.030 & 0.410 & 0.583 & 1.000 & -0.173 & 0.083 \\
& Male & 0.057 & -0.024 & -0.006 & -0.173 & 1.000 & 0.553 \\
\multirow{2}{*}{ US } & Female & -0.175 & -0.097 & 0.036 & 0.083 & 0.553 & 1.000 \\
\hline
\end{tabular}

(b)

\begin{tabular}{cccccccc}
\hline & & \multicolumn{7}{c}{ Principle components } \\
\hline \multirow{3}{*}{ Can } & & 1 & 2 & 3 & 4 & 5 & 6 \\
& Male & -0.351 & -0.603 & -0.315 & 0.205 & 0.160 & 0.588 \\
& Female & -0.458 & -0.460 & -0.128 & -0.272 & -0.101 & -0.691 \\
\multirow{3}{*}{ GBR } & Male & 0.446 & -0.220 & -0.291 & -0.676 & -0.399 & 0.229 \\
& Female & 0.472 & -0.087 & -0.513 & 0.082 & 0.646 & -0.286 \\
& Male & -0.248 & 0.462 & -0.731 & 0.213 & -0.380 & -0.036 \\
& Female & -0.429 & 0.394 & -0.050 & -0.613 & 0.493 & 0.200 \\
& eigenvalue & 2.747 & 1.310 & 0.906 & 0.566 & 0.288 & 0.183 \\
& $\%$ & $45.8 \%$ & $21.8 \%$ & $15.1 \%$ & $9.4 \%$ & $4.8 \%$ & $3.1 \%$ \\
& cum $\%$ & $45.8 \%$ & $67.6 \%$ & $82.7 \%$ & $92.2 \%$ & $96.9 \%$ & $100.0 \%$ \\
\hline
\end{tabular}

\section{State Transition probabilities}

These indicate probabilities of staying in the same state which in turn affect the average length of stay in the state (see Table 5).

In a Hidden Markov Model it is possible to compute the probability of being in either state 1 or state 2 given the data. The graph (Figure 3 ) shows the posterior probabilities of State 2 .

\section{Discussion}

The goal of this paper was to contribute to providing a long-term perspective regarding suicide across three Western countries (Canada, Great Britain and the US). Long term focuses on statistics of suicide tend to report only descriptive trends over time periods. The sixty-year time period covered in this study was deemed sufficient to uncover any underlying useful trends in the data. Preliminary descriptive analysis of the data shows (consistent with other studies) a higher suicide rate for males than females in all three countries. Our use of Hidden Markov Models to examine time trends in suicide rates of males and females in the 3 countries uncovered 2 long term states underlying the data. In state 1 
Table 5. Transition matrix for state probabilities.

\begin{tabular}{cccc}
\hline & \multicolumn{3}{c}{ Transition matrix } \\
\hline & to State 1 & To State 2 & Length of stay in State \\
\hline From State 1 & 0.921 & 0.079 & $12.61 \mathrm{yrs}$ \\
From State 2 & 0.039 & 0.961 & $25.39 \mathrm{yrs}$ \\
\hline
\end{tabular}

Posterior Probabilities of State 2

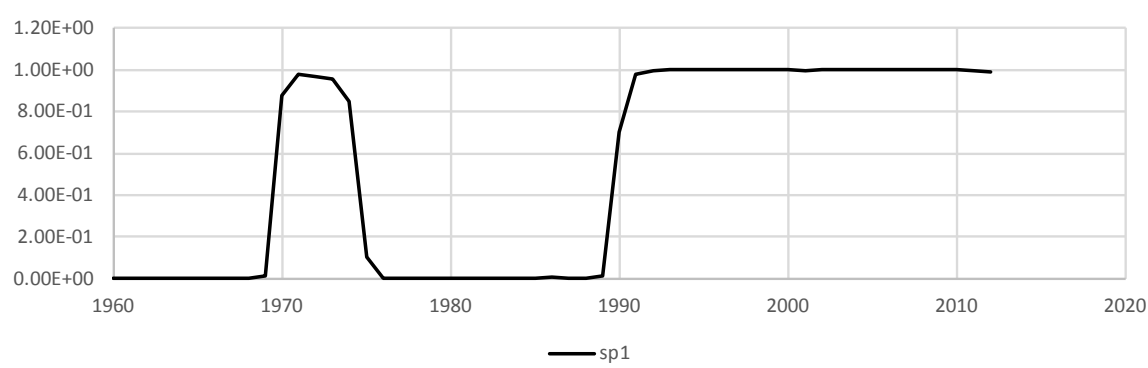

Figure 3. State 2 posterior probabilities.

(25.4 year) suicide rates are drifting upwards for Canadian males and females while drifting downwards for males and females in both Great Britain and the US. When in state 2 (12.6 years), suicide rates are drifting downwards for all groups except Canadian and US females. It is unclear to the authors what these patterns suggest in explanation. Further research could explore the social reasons (perhaps the social policies and economic health of the countries) for these trends during the two different states, and whether they hold in other countries. It would also be worth exploring if these underlying trends are found in other countries.

It may also be useful to point out that Hidden Markov Models, used to uncover hidden underlying trends in suicide in this article, may have a wider role to play in the emerging field of Cliodynamics, which studies historical trends in order to predict future periods of social and economic stability or instability [13] [14].

\section{Conflicts of Interest}

The authors declare no conflicts of interest regarding the publication of this paper.

\section{References}

[1] World Health Organization (WHO) (2018) Suicide. https://www.who.int/news-room/fact-sheets/detail/suicide

[2] Milner, A., Spittal, M.J., Pirkis, J. and Lamontagne, A.D. (2013) Suicide by Occupation: Systematic Review and Meta-Analysis. British Journal of Psychiatry, 203, 409-416. https://doi.org/10.1192/bjp.bp.113.128405

[3] Milner, A., Witt, K., LaMontagne, A.D. and Niedhammer, I. (2018) Psychosocial Job Stressors and Suicidality: A Meta-Analysis and Systematic Review. Occupational 
Environmental Medicine, 75, 245-253. https://doi.org/10.1136/oemed-2017-104531

[4] Blacker, C.J., Lewis, C.P., Cosima, C.C., Bostwick, M.J. and Rackey, S.J. (2019) Medical Student Suicide Rates: A Systematic Review of the Historical and International Literature. Academic Medicine, 94, 274-280.

https://doi.org/10.1097/ACM.0000000000002430

[5] Office for National Statistics (UK) (2018) Suicide in the UK: 2017 Registrations. https://www.ons.gov.uk/peoplepopulationandcommunity/birthsdeathsandmarriage s/deaths/bulletins/suicidesintheunitedkingdom/2017registrations

[6] Samaritans UK (2019) Suicide: Facts and Figures.

https://www.samaritans.org/about-us/our-research/facts-and-figures-about-suicide

[7] Canadian Association for Suicide Prevention (CASP). https://suicideprevention.ca

[8] Findlay, L. (2017) Depression and Suicidal Ideation among Canadians Aged 15-24. Health Reports (Statistics Canada), 28, 3-11

https://www150.statcan.gc.ca/n1/pub/82-003-x/2017001/article/14697-eng.htm

[9] American Foundation for Suicide Prevention (AFSP). https://afsp.org/about-suicide/suicide-statistics

[10] Hedegaard, H., et al. (2018) Suicide Rates in the United States Continue to Increase. NCHS Data Brief No. 309, National Center for Health Statistics, Hyattsville. http://www.cdc.gov/nchs/products/databriefs/db309.htm

[11] Organization for Economic Co-Operation and Development (OECD). https://data.oecd.org/healthstat/suicide-rates.htm

[12] Wilks, S.S. (1938) The Large-Sample Distribution of the Likelihood Ratio for Testing Composite Hypotheses. The Annals of Mathematical Statistics, 9, 60-62. https://doi.org/10.1214/aoms/1177732360

[13] Turchin, P. (2019) Cliodynamics: History as Science. http://peterturchin.com/cliodynamics

[14] Turchin, P. (2008) Arise "Cliodynamics". Nature, 454, 34-35. https://doi.org/10.1038/454034a 